\title{
Transcriptomic changes in human renal proximal tubular cells revealed under hypoxic conditions by RNA sequencing
}

\author{
WENMIN YU, YIPING LI, ZHI WANG, LEI LIU, JING LIU, FENGAN DING, XIAOYI ZHANG, \\ ZHENGYUAN CHENG, PINGSHENG CHEN and JUN DOU
}

Medical School of Southeast University, Nanjing, Jiangsu 210009, P.R. China

Received November 24, 2015; Accepted July 7, 2016

DOI: $10.3892 / \mathrm{ijmm} .2016 .2677$

\begin{abstract}
Chronic hypoxia often occurs among patients with chronic kidney disease (CKD). Renal proximal tubular cells may be the primary target of a hypoxic insult. However, the underlying transcriptional mechanisms remain undefined. In this study, we revealed the global changes in gene expression in HK-2 human renal proximal tubular cells under hypoxic and normoxic conditions. We analyzed the transcriptome of $\mathrm{HK}-2$ cells exposed to hypoxia for $24 \mathrm{~h}$ using RNA sequencing. A total of 279 differentially expressed genes was examined, as these genes could potentially explain the differences in HK-2 cells between hypoxic and normoxic conditions. Moreover, 17 genes were validated by qPCR, and the results were highly concordant with the RNA seqencing results. Gene Ontology and Kyoto Encyclopedia of Genes and Genomes (KEGG) pathway enrichment analyses were performed to better understand the functions of these differentially expressed genes. The upregulated genes appeared to be significantly enriched in the pathyway of extracellular matrix (ECM)-receptor interaction, and in paticular, the pathway of renal cell carcinoma was upregulated under hypoxic conditions. The downregulated genes were enriched in the signaling pathway related to antigen processing and presentation; however, the pathway of glutathione metabolism was downregulated. Our analysis revealed numerous novel transcripts and alternative splicing events. Simultaneously, we also identified a large number of single nucleotide polymorphisms, which will be a rich resource for future marker development. On the whole, our data indicate that transcriptome analysis provides valuable information for a
\end{abstract}

Correspondence to: Professor Pingsheng Chen or Professor Jun Dou, Medical School of Southeast University, 87 Ding Jiaqiao Road, Nanjing, Jiangsu 210009, P.R. China

E-mail: chenpsh@sina.com

E-mail: njdoujun@seu.edu.cn

Abbreviations: CKD, chronic kidney disease; DEGs, differentially expressed genes; ECM, extracellular matrix

Key words: transcriptomic changes, HK-2 cells, hypoxia, sequencing more in depth understanding of the molecular mechanisms in CKD and renal cell carcinoma.

\section{Introduction}

Chronic kidney disease (CKD) is increasingly recognized as a worldwide public health issue $(1,2)$, and chronic hypoxia often occurs among patients with CKD. Renal hypoxia is emerging as a key player by influencing tubular epithelial cells during the process of the acute kidney injury (AKI)-to-CKD transition. Under hypoxia, these injured cells fail to redifferentiate, which results in aggravating hypoxia and gives rise to a vicious circle (3).

Some studies have shown that human renal proximal tubular cells (namely HK-2 cells) may be the primary target of a hypoxic insult (4). Although it is clear that HK-2 cells demand high concentrations of oxygen to maintain their metabolic functions (5-7), the relevant mechanisms have not yet been identified. Hypoxia can regulate the expression of a wide variety of genes that may be induced or suppressed by transcriptional or post-transcriptional mechanisms (8-10). Hypoxia response elements have been identified in the regulatory regions of a number of genes and contain consensus binding sites for the transcription factor hypoxia-inducible factor- $1 \alpha(\mathrm{HIF}-1 \alpha)$ (11-13). Several studies have demonstrated changes in collagen gene expression in various mesenchymal cells exposed to hypoxia (14-17).

Renal proximal tubular cells are a primary target for hypoxic injury in the kidneys and some studies have reported a distinct cohort of 48 genes with a closely shared hypoxia-dependent expression profile, identified by microarray analysis $(18,19)$. Since the global transcriptional mechanisms underlying these events are not yet fully understood, in this study, we undertook an unbiased approach using RNA sequencing (RNA-Seq) to define the transcriptomic responses regulated by hypoxia in HK-2 cells. RNA sequencing technology provides major advantages over microarray technology, providing more precise information on absolute transcript levels and transcript variants, and currently unannotated transcribed regions can be analyzed; therefore, it is increasingly used in various biological applications (20). However, to date, and to the best of our knowledge, no RNA-seq assays demonstrating transcriptomic changes have been reported in HK-2 cells under hypoxic conditions. 
In this study, using RNA-seq analysis, we examined HK-2 cells under hypoxic and normoxic conditions to clarify global changes in gene expression under such conditions. The expression levels of selected genes were further validated by reverse transcription-quantitative PCR (RT-qPCR). Although the expression levels of the vast majority of genes remained unaltered, a specific cluster of genes with a closely shared expression profile were regulated undr hypoxic conditions. By using stringent bioinformatics analysis of the data, our findings extend those of previous and related publications on gene expression patterns that may be critical for the development of CKD.

\section{Materials and methods}

Cell culture and exposure to hypoxia. The HK-2 cell line was purchased from the Cell Bank of Type Culture Collection of Chinese Academy of Sciences (Shanghai, China). The HK-2 cells were cultured in high-glucose DMEM supplemented with $10 \%$ fetal bovine serum (FBS), $100 \mathrm{U} / \mathrm{ml}$ penicillin and $100 \mu \mathrm{g} / \mathrm{ml}$ streptomycin (both from Thermo Fisher Scientific, Waltham, MA, USA) in humidified air containing $5 \% \mathrm{CO}_{2}$ at $37^{\circ} \mathrm{C}$. After the cells doubled and redoubled, the viability of the cells was at its maximum, and this phase can be identified as the logarithmic growth phase. Cells in the logarithmic growth phase were used in all of the experiments.

The cells were seeded in 6-well plates at a density of $5 \times 10^{5}$ cells/well and cultured for $24 \mathrm{~h}$. To mimic hypoxic conditions, the cells in medium were then incubated under low oxygen conditions. The oxygen concentrations were maintained at $1-3 \%$ using a compact gas oxygen controller, which was held under positive pressure in an atmosphere of 94-92\% $\mathrm{N}_{2} / 5 \% \mathrm{CO}_{2} / 1-3 \% \mathrm{O}_{2}$ for $24 \mathrm{~h}$. The control cells were cultured under normoxic conditions $\left(5 \% \mathrm{CO}_{2}\right.$ for $\left.24 \mathrm{~h}\right)$.

Western blot analysis. The cells were plated in 24-well plates and cultured in DMEM supplemented with $10 \%$ FBS for $24 \mathrm{~h}$. Following exposure to hypoxia for $24 \mathrm{~h}$, the cells were lysed with immunoprecipitation assay buffer containing protease inhibitors. The cell lysates were centrifuged at $14,000 \mathrm{x} \mathrm{g}$ for $10 \mathrm{~min}$ at $4^{\circ} \mathrm{C}$ followed by incubation on ice. The protein concentrations of the lysates were examined using the Bradford protein assay kit (Vazyme Biotech Inc., Nanjing, China). The cell lysates were boiled and separated by sodium dodecyl sulfate polyacrylamide gel electrophoresis and transferred onto polyvinylidene difluoride membranes via semi-dry transfer (Bio-Rad Laboratories Inc., Hercules, CA, USA). The membranes were washed in Tris-buffered saline containing $0.1 \%$ Tween-20 (TBST), blocked with 5\% non-fat milk in TBST for $1 \mathrm{~h}$ at room temperature, and incubated with a primary rabbit monoclonal antibody against HIF-1 $\alpha$ (Cat. no. ab179483; dilution 1:5,000; Abcam, Cambridge, UK) or glyceraldehyde-3-phosphate dehydrogenase (GAPDH) antibody (Cat. no. 10494; dilution 1:5,000; Proteintech Technology, Inc., Wuhan, China) overnight at $4^{\circ} \mathrm{C}$. The membranes were washed 3 times in TBST, followed by incubation with the appropriate horseradish peroxidase-linked secondary anti-rabbit antibodies (Cat. no. SA00001-2; dilution 1:5,000; Proteintech Technology, Inc.) for $1 \mathrm{~h}$ at room temperature. The specific proteins on the blots were developed by enhanced chemiluminescence (ECL; Vazyme Biotech, Inc.) and visualized as the bands on the CL-XPosure Film (Thermo Fisher Scientific). The optical densities of the bands were measured on the GS710 Densitometer and analyzed using Quantity One image analysis software (Bio-Rad Laboratories Inc.).

RNA extraction and RNA-Seq analysis. Total RNA was isolated from cells using the TRIzol reagent (Invitrogen, Carlsbad, CA, USA) according to the manufacturer's instructions. Total RNA sample quality and integrity were controlled using a Bioanalyzer 2100 (Agilent, Boeblingen, Germany). For library preparation, $5 \mu \mathrm{g}$ total RNA were captured by Dynabeads Oligo(dT) sheared to fragements of $\sim 200 \mathrm{bp}$, and reverse transcribed using the SuperScript III cDNA Synthesis kit, as previously described (21) (both from Life Technologies, Grand Island, NY, USA). cDNA was end-repaired, A-tailed and ligated to Illumina sequencing adapters and amplified by PCR. Library preparation were performed using the TruSeq RNA LT V2 Sample Prep kit, as previously described (22) (Illumina, San Diego, CA, USA). The sequencing library was qualified by Qubit 2.0 (Life Technologies) and Bioanalyzer 2100 (Agilent), then sequenced on a Illumina HiSeq 2000 with $2 \times 100 \mathrm{bp}$ paired-end sequencing, which were controlled by HiSeq Control software. Raw reads in FASTQ format were aligned to the human genome (GRCh37/hg19) using TopHat software 2.0.10, as previously described (23). This parameter defines -G genes.gtf -r 0--mate-std-dev 80--solexa1.3-qual. Transcript isoform assembly and abundance estimation were performed using Cufflinks (v1.3.0) (24) and combined with gene annotations from the National Center for Biotechnology Information (ftp://igenome:G3nom3s4u@ussd-ftp.illumina. com/Homo_sapiens/UCSC/hg19/Homo_sapiens_UCSC_hg19. tar.gz). The cDNA libraries were sequenced using the HiSeq $^{\mathrm{TM}} 2000$ sequencing platform (Illumina).

Validation by $q P C R$. qPCR were executed to validate the data obtained by RNA-seq analysis. Total RNA was isolated from the HK-2 cells using the RNeasy Mini kit and an RNase-free DNase set (both from Vazyme Biotech, Inc.). qPCR was performed to determine the expression of genes in a SYBR-Green PCR Master mix (Vazyme Biotech, Inc.) using the StepOnePlus ${ }^{\mathrm{TM}}$ realtime PCR detection system (Step One Plus 2.1 software) with universal thermal cycling parameters. The primer sequences are listed in Table I. GAPDH was used as an internal control for target genes for reaction efficiency. The $\Delta \Delta \mathrm{Ct}$ method was used to determine the relative amounts of product.

Gene Ontology (GO) and pathway analysis for differentially expressed genes (DEGs). GO functional classifications were analyzed at the macroscopic level based on cellular component, biological process and molecular function categories. GO terms with a $\mathrm{P}<0.01$ by Fisher's exact test were considered enriched. Pathway analysis was determined by the DEGs according to the KEGG, BioCarta and Reactome databases. Significant pathways were selected in accordance with Fisher's exact test followed by Benjamini Hochberg multiple testing correction, and the threshold of significance was defined as a value of $\mathrm{P}<0.05$.

Identification of alternative splicing (AS) events and the discovery of novel mRNA transcripts. Mixture of isoforms (MISO) 
Table I. Primers used in qPCR.

\begin{tabular}{|c|c|c|}
\hline $\begin{array}{l}\text { Gene } \\
\text { name }\end{array}$ & Primer sequences & $\begin{array}{c}\text { Lengtl } \\
\text { (bp) }\end{array}$ \\
\hline DL4A2 & $\begin{array}{l}\text { F: 5'-TGCTACCCGGAGAAAGGAG-3' } \\
\text { R: 5'-CTTTGCGGCCCTGTAGTCC-3' }\end{array}$ & 106 \\
\hline OL7A1 & $\begin{array}{l}\text { F: 5'-CCAGAGGTCGTTCCGGAG-3' } \\
\text { R: 5'-GCTCTTCCCACTTCGACC-3' }\end{array}$ & 81 \\
\hline THBS3 & $\begin{array}{l}\text { F: 5'-ACAGTTCTCCTGCGACTCCG-3' } \\
\text { R: 5'-GCATCCTCAAATACGCCTTC-3' }\end{array}$ & 172 \\
\hline AMA5 & $\begin{array}{l}\text { F: 5'-ACCCAAGGACCCACCTGTAG-3' } \\
\text { R: 5'-TCATGTGTGCGTAGCCTCTC-3' }\end{array}$ & 169 \\
\hline AMB1 & $\begin{array}{l}\text { F: 5'-TGGCTGAAGTGGAACAGCTCTC-3' } \\
\text { R: 5'-TGTCTTCAACAGAATGTCTTCAGCA-3' }\end{array}$ & 95 \\
\hline TGB4 & $\begin{array}{l}\text { F: 5'-AGAGGGAGGAAGAGGATGGC-3' } \\
\text { R: 5'-GCAGTAGGCGCAGTCCTTAT-3' }\end{array}$ & 168 \\
\hline MUC1 & $\begin{array}{l}\text { F: 5'-CGTCATGGACATTGATGGTACC-3' } \\
\text { R: 5'-GGTACCTCCTCTCACCTCCTCCAA-3' }\end{array}$ & 228 \\
\hline VEGF & $\begin{array}{l}\text { F: 5'-GAGTACATCTTCAAGCCATCCTG-3' } \\
\text { R: 5'-TGCTCTATCTTTCTTTGGTCTGC-3' }\end{array}$ & 203 \\
\hline LUT1 & $\begin{array}{l}\text { F: 5'-AAGGTGATCGAGGAGTTCTACA-3' } \\
\text { R: 5'-ATGCCCCCAACAGAAAAGATG-3' }\end{array}$ & 119 \\
\hline LIN2 & $\begin{array}{l}\text { F: 5'-TGAGATGGCAGAGAACGGTGTGAA-3' } \\
\text { R: 5'-TTGCGGCTCTAGCTTCTGGATGAT-3' }\end{array}$ & 84 \\
\hline TGF- $\beta 1$ & $\begin{array}{l}\text { F: 5'-GAGCCTGAGGCCGACTACTA-3' } \\
\text { R: 5'-CGGAGCTCTGATGTGTTGAA-3' }\end{array}$ & 130 \\
\hline DGFB & $\begin{array}{l}\text { F: 5'-TGATGCCGAGGAACTATTCATCT-3' } \\
\text { R: 5'-TTTCTTCTCGTGCAGTGTCAC-3' }\end{array}$ & 178 \\
\hline HIG2 & $\begin{array}{l}\text { F: 5'-CCACAGTGCAAGACTCCATC-3' } \\
\text { R: 5'-GCCATACTGCTGAGGAAAGC-3' }\end{array}$ & 150 \\
\hline BNIP3L & $\begin{array}{l}\text { F: 5'-TCGAGCCGCCGCCGCCCCTG-3' } \\
\text { R: 5'-CATTGCCATTATCATTGCCATTG-3' }\end{array}$ & 138 \\
\hline JUNB & $\begin{array}{l}\text { F: 5'-GTCACCGAGGAGCAGGAGG-3' } \\
\text { R: 5'-TCTTGTGCAGATCGTCCAGG-3' }\end{array}$ & 63 \\
\hline MMP-1 & $\begin{array}{l}\text { F: 5'-GATGAAGTCCGGTTTTTCAAAG-3' } \\
\text { R: 5'-GGGGTATCCGTGTAGCACCAT-3' }\end{array}$ & 71 \\
\hline GLUT3 & $\begin{array}{l}\text { F: 5'-TTCGTCTCTAGCCTGCACTG-3' } \\
\text { R: 5'-ACACAACTTCTCCGGGTGAC-3' }\end{array}$ & 79 \\
\hline GAPDH & $\begin{array}{l}\text { F: 5'-GGAAGGTGAAGGTCGGAGTCA-3' } \\
\text { R: 5'-GCAACAATATCCACTTTACCAGAGTT }\end{array}$ & $\begin{array}{l}90 \\
3^{\prime}\end{array}$ \\
\hline
\end{tabular}

F, forward; R, reverse.

analysis (25) was used to examine differentially regulated exons across samples. MISO analysis was accomplished using pairedend reads according to the workflow given (25). The reads alignment files produced by TopHat and the pre-build human genome alternative events and were downloaded from the MISO reference manual page (http://genes.mit.edu/burgelab/miso/ docs/\#gff-event-annotation); the date of access for the databases was April 30, 2015.
Identification of single nucleotide polymorphisms (SNPS). The identification of SNPs was successively performed using the mutation detection software GATK, as previously described $(26,27)$.

\section{Results}

Expression of HIF-1 $\alpha$ in HK-2 cells under hypoxic conditions. The HK-2 cells were exposed to the hypoxic milieu $\left(1 \% \mathrm{O}_{2}\right.$ for $24 \mathrm{~h}$ ) to examine the effects of oxygen deprivation on HK-2 cells, and the expression of HIF-1 $\alpha$ was examined by western blot analysis. The results revealed that hypoxia upregulated the expression level of HIF-1 $\alpha$ (Fig. 1A and B).

Illumina sequencing and overview of the sequence reads. We obtained a global overview of the transcriptome from the two cDNA libraries separately constructed from HK-2 cells under hypoxic and normoxic conditions by using the Illumina $\mathrm{HiSeq}^{\mathrm{TM}} 2000$ sequencing platform. The two libraries were named after the hypoxic group and normoxic group, respectively. A total of $111,829,528$ raw reads was obtained from the cDNA libraries. After the raw reads were filtered, a total of $93,002,921$ high-quality mappable reads remained, of these, $51,182,720$ reads were from cells under hypoxic conditions and 41,820,201 reads were from cells under normoxic conditions. The sequencing reads were mapped to the human genome (GRCh37/hg19); at least $86 \%$ of the sequence bases could be aligned to the genome, and $>77 \%$ of the mapped read bases were localized in mRNA regions. In addition, the Q20 (those reads with an average quality score $>20$ ) was $>96 \%$, and the GC content was consistently $\sim 53 \%$ for both hypoxic and normoxic conditions, which suggested that the sequencing was highly accurate.

An overview of the sequencing process is presented in Table II. Of the total number of clean reads, $87.06 \%$ mapped to multiple $(1.69 \%)$ or unique $(85.92 \%)$ genome locations for hypoxic conditions, and $86.89 \%$ mapped to multiple $(1.79 \%)$ or unique $(85.11 \%)$ genome locations for normoxic conditions. Thus, the read mapping evenness across the transcripts of two samples was good. Volcano plots in the magnitude of gene expression ratios are displayed in Fig. 1C and demonstrate the significance of differences in gene expression between the two samples.

Evaluation of DEGs. To examine the differences in gene expression patterns between the hypoxic group and normoxic group, the criteria for the detection of differential expression gene in this study was the absolute value of fold change $>2$ and a higher statistical significance value (P-value $<0.05)$. A total of 279 genes was observed to be differentially expressed between the hypoxic group and normoxic group (data not shown). Of these genes, 201 were specifically upregulated, and 78 genes were markedly downregulated in the hypoxic group vs. the normoxic group (data not shown). These results demonstrated that the number of upregulated DEGs was considerably higher than the number of downregulated DEGs. Of the 201 upregulated genes, it is notable that a host of genes are associated with hypoxia, such as encode transforming growth factor (TGF)- $\beta 1$, vascular endothelial cell growth factor (VEGF), 6-phosphofructo2-kinase/fructose-2,6-biphosphatase 4 protein (PFKFB4) and 
A
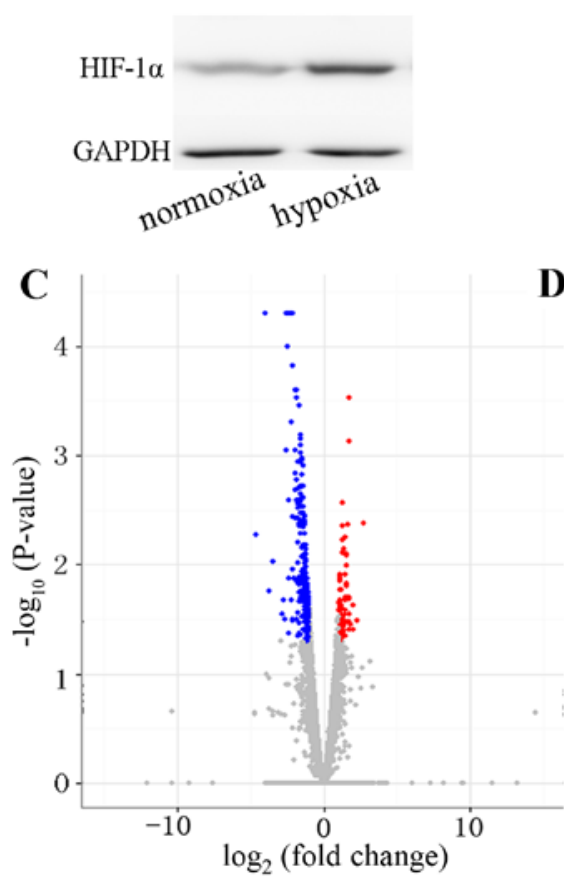

D
B
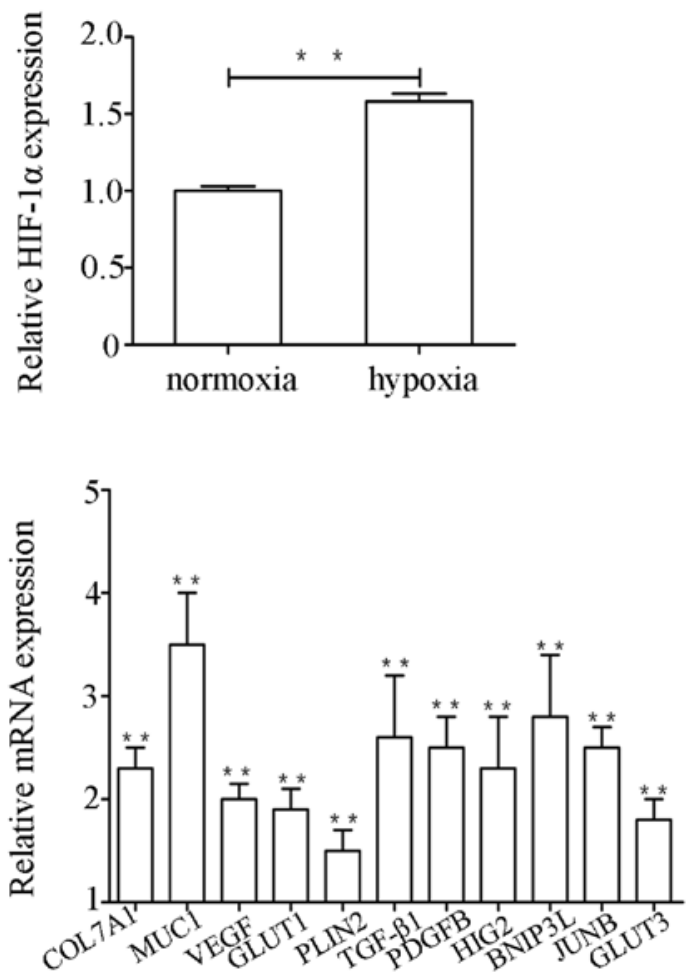

Figure 1. Expression of hypoxia-inducible factor-1 $\alpha(\mathrm{HIF}-1 \alpha)$ in human renal proximal tubular cells (HK-2) cells under hypoxic conditions and Volcano plots in the magnitude of gene expression ratios. (A) Western blot analysis of HIF-1 $\alpha$ protein. (B) Ratios of the expression of HIF-1 $\alpha$ relative to that of GAPDH. Hypoxia involved exposure of the cells to $1 \% \mathrm{O}_{2}$ for $24 \mathrm{~h}$. (C) Volcano plot of differentially expressed genes in HK-2 cells under normoxic and hypoxic conditions. The $\log _{2}$ fold change difference between the hypoxic samples and normoxic samples is represented on the x-axis, and the negative log of P-values is represented on the $\mathrm{y}$-axis. The differentially expressed genes were plotted in blue for the upregulated genes and red for downregulated genes, and non-significant genes are shown as gray points. (D) Validation of selected genes based on RNA-Seq were verified using qPCR. The relative expression levels of differentially expressed genes are shown (relative value to that of normoxia, value of normoxia assigned values of 1 ). Results were analyzed by an unpaired Student's t-test, ${ }^{* *} \mathrm{P}<0.01$.

Table II. Summary of clean Illumina RNA-seq reads and Illumina transcriptome sequencing in HK-2 cells under hypoxic and normoxic conditions.

\begin{tabular}{lll}
\hline Reads category & \multicolumn{1}{c}{$\begin{array}{c}\text { Hypoxic } \\
\text { HK-2 cells }\end{array}$} & \multicolumn{1}{c}{$\begin{array}{c}\text { Normoxic } \\
\text { HK-2 cells }\end{array}$} \\
\hline Total raw reads & $61,284,796$ & $50,544,732$ \\
Total clean reads & $58,425,234$ & $48,128,048$ \\
Total mapped reads & $51,182,720(87.06 \%)$ & $41,820,201(86.89 \%)$ \\
Multiple mapped reads & $984,919(1.69 \%)$ & $859,998(1.79 \%)$ \\
Uniquely mapped reads & $50,197,801(85.92 \%)$ & $40,960,203(85.11 \%)$ \\
Q20 (\%) & $96.70(\%)$ & $96.92(\%)$ \\
GC content (\%) & $53.29(\%)$ & $53.29(\%)$ \\
\hline
\end{tabular}

HK-2, human renal proximal tubular cells.

glucose transporter protein (GLUT1). The above-mentioned data also demonstrated that the result of RNA-Seq in this study was reliable.

Validation of gene expression. A total of 17 genes was selected and quantified to verify the RNA-Seq data by qPCR. As shown in Figs. 1D and 4B, all of the 17 genes were confirmed.
The results illustrated that the expression patterns of these genes were highly consistent with the RNA-Seq data, which suggested that our transcriptome analysis was reliable.

GO and KEGG pathway functional enrichment analysis of the DEGs. GO enrichment and pathway analysis of the DEGs were accomplished to determine the potential biological functions of the DEGs. The enriched GO terms are shown in Fig. 2 according to 3 top-level ontologies, namely biological process (BP), cellular component (CC) and molecular function $(\mathrm{MF})$. With respect to biological processes, the upregulated genes were mainly enriched for extracellular matrix metabolism, extracellular structure organization and locomotion, and the downregulated genes were mostly related to ventricular cardiac muscle cell differentiation, regulation of cellular amino acid metabolic process and the positive regulation of cardiac muscle contraction. For molecular function, the upregulated genes were invovled in protein binding, glycoprotein binding and growth factor binding, and the downregulated genes were associated with diphosphotransferase activity, growth factor activity and MHC II protein complex binding. According to the cellular component annotation, the upregulated genes were mainly localized in the extracellular matrix (ECM) including proteinaceous ECM and basement membrane, while the downregulated genes were enriched in the cytoplasm, macro- 

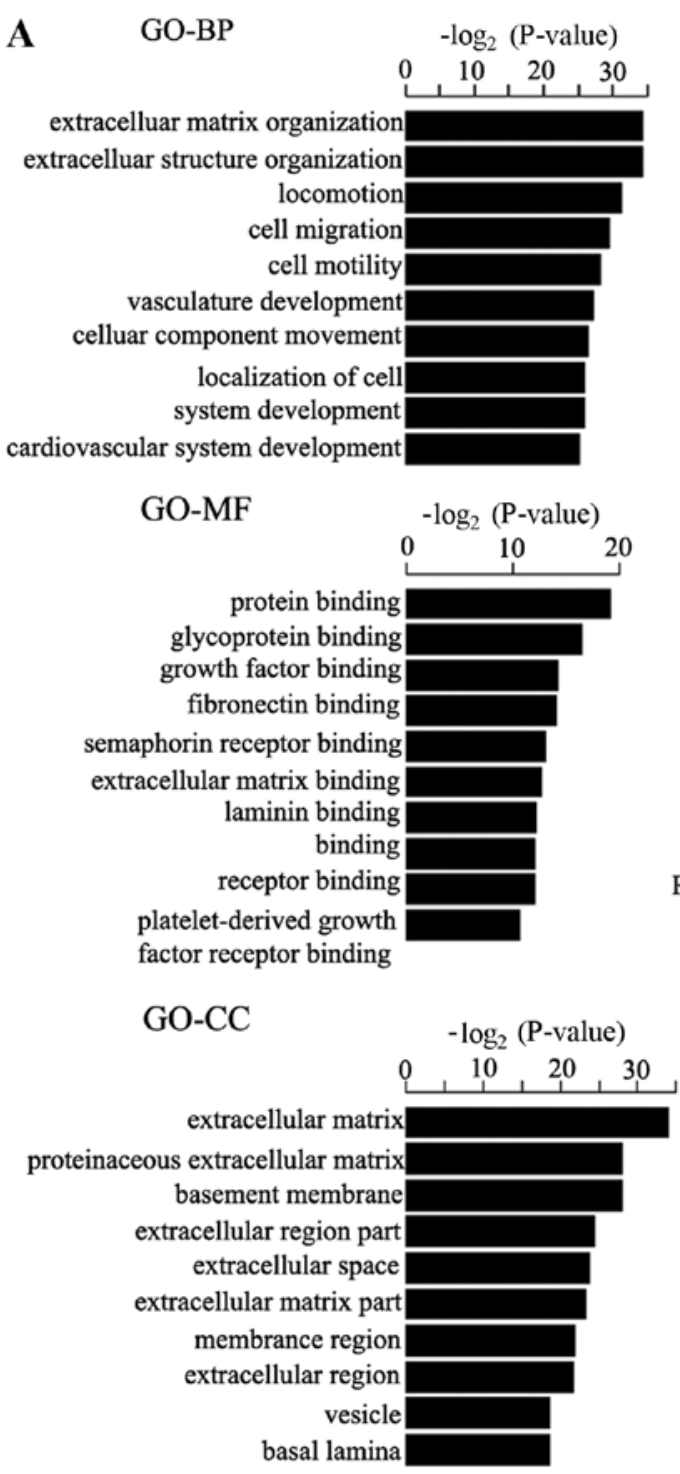

B B
ventricular cardiac muscle cell differentiation
position of cellular amino acid metabolic process
regulation of cellular amine metabolic process
positive regulation of striated muscle contraction
cullin deneddylation
protein deneddylation
polyamine biosynthetic process
regulation of tissue remodeling

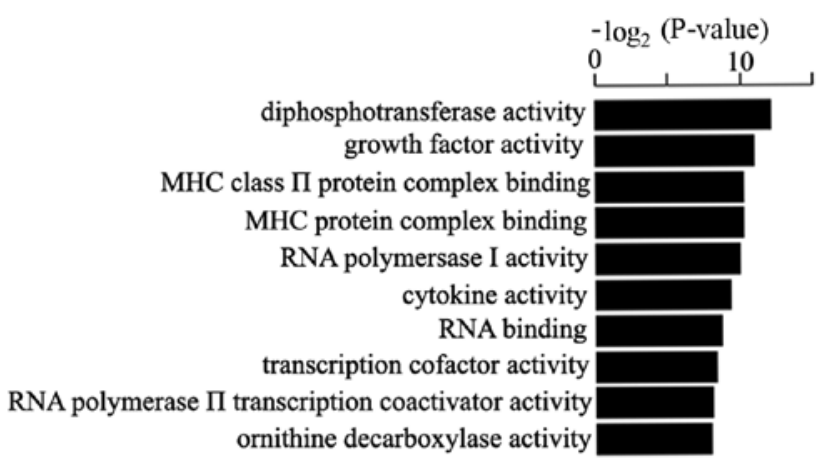

RNA polymerase $\Pi$ transcription coactivator activity ornithine decarboxylase activity

Figure 2. Gene Ontology (GO) analysis of differentially expressed genes (DEGs) in human renal proximal tubular cells (HK-2) cells under hypoxic and normoxic conditions. The top 10 (A) upregulated and (B) downregulated GO terms for biological processes, molecular function and cellular component were generated by a bar plot with mostly significant P-values. BP, biological process; CC, cellular component and MF, molecular function.

molecular complex and DNA-directed RNA polymerase I complex.

For KEGG pathway enrichment analysis, the up- and downregulated genes appeared to be significantly enriched in ECM-receptor interaction (Figs. 3 and 4A) and antigen processing and presentation, respectively. This result is consistent with that of the enriched biological process, and pathway-based analyses help to further understand the biological functions of genes. The 11 DEGs involved in the ECM-receptor interaction pathway included collagen type IV alpha 2 (COL4A2), heparan sulfate proteoglycan 2 (HSPG2), integrin subunit alpha 3 (ITGA3), integrin subunit beta 3(ITGB3), integrin subunit beta4(ITGB4), integrin subunit beta 8 (ITGB8), laminin subunit alpha 3 (LAMA3), laminin subunit alpha 5 (LAMA5), laminin subunit beta 1 (LAMB1), thrombospondin 3 (THBS3) and agrin (AGRN); some of these genes were confirmed by qPCR (Fig. 4B).

Among the upregulated genes, platelet derived growth factor subunit B (PDGFB), solute carrier family 2 member 1 (SLC2A1),
TGFA, TGF- $\beta 1$ and VEGFA were enriched in the pathway of renal cell carcinoma (RCC). Furthermore, PDGFB, TGF- $\beta 1$ and VEGFA were validated by qPCR. These genes dispalyed differential expression patterns in the HK-2 cells under hypoxic conditions. Of the downregulated genes, ornithine decarboxylase 1 (ODC1) and spermidine synthase (SRM) were enriched in the pathway of glutathione metabolism. In brief, the DEGs provide a better understanding of gene expression patterns in HK-2 cells under hypoxic conditions.

Identification of AS events and the discovery of novel mRNA transcripts. In this study, we obtained a complete picture of AS events between the hypoxic group and normoxic group by MISO (25). All theoretical splicing junctions were identified, and a total of 51,387 and 49,144 AS events were found in the hypoxic group and normoxic group, respectively. In this study, AS events identified contain 5 different types: alternative 5 ' splice site (A5SS), alternative 3' splice site (A3SS), exon skipped (ES), intron retention (IR) and mutually exclusive 
A

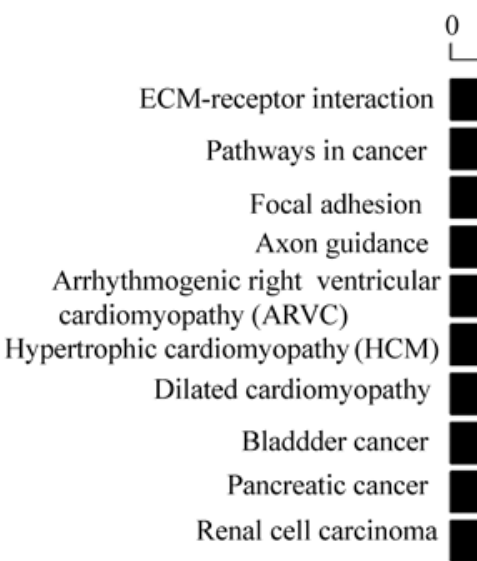

- $\log _{2}$ (P-value)

$\begin{array}{llll}0 & 10 & 20 & 30\end{array}$
B

Antigen processing and presention

Protein processing in endoplasmic reticulum

Thiamine metabolism

Proteasome

Spliceosome

Glutathione metabolism

Arginine and proline metabolism

NOD-like receptor signaling pathway

Purine metabolism $-\log _{2}$ (P-value)

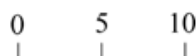

Figure 3. KEGG pathway analysis of differentially expressed genes (DEGs) in human renal proximal tubular cells (HK-2) cells under hypoxic and normoxic conditions. (A and B) The top 10 upregulated and downregulated pathways, respectively. The data shown are the negative $\log _{2}$ P-values within each category.

A

ECM-RECEPTOR INTERACTION

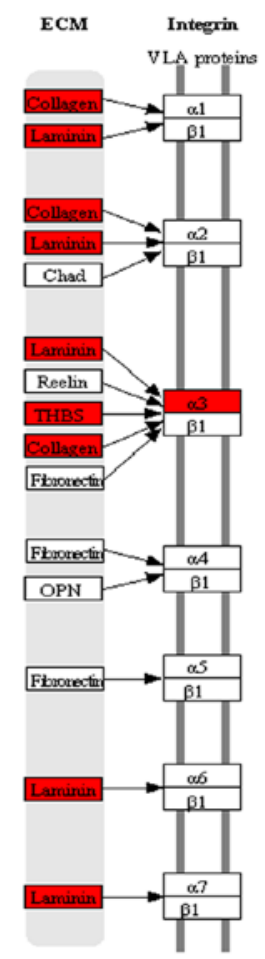

ECM

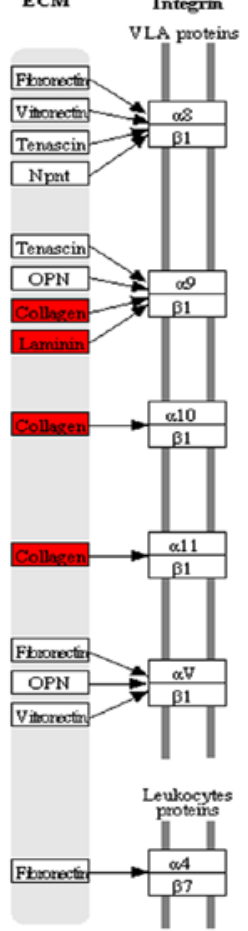

ECM
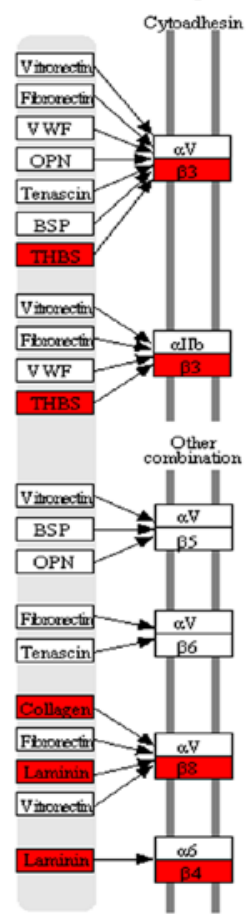

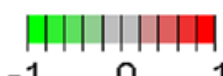

$-1$

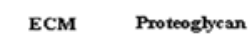

ECM Proteoglyean
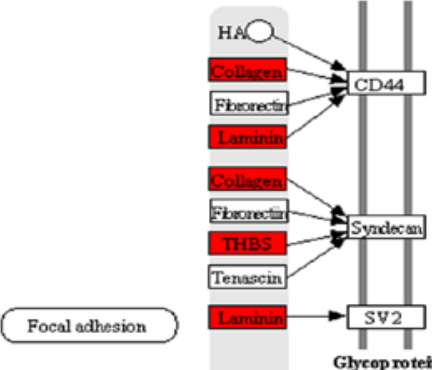

Fiboreter
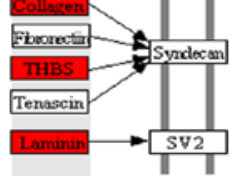
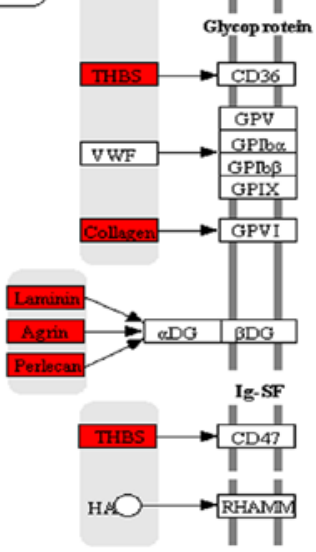

B

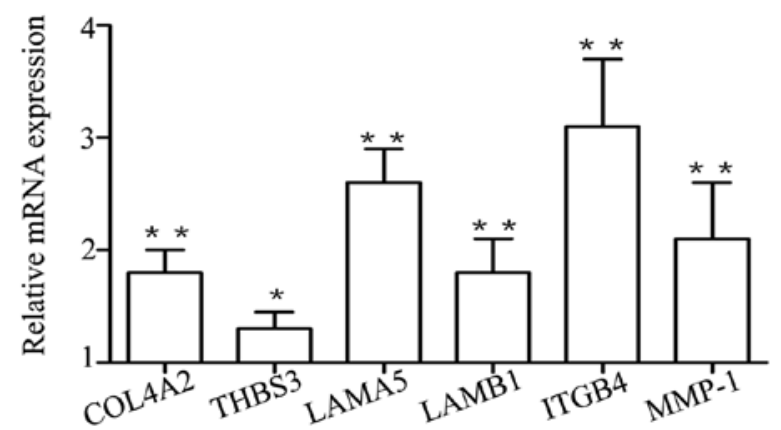

Figure 4. Extracellular matrix (ECM)-receptor interaction pathway. (A) Upregulated genes are colored in red. (B) Expressions of the COL4A2, ITGB4, LAMA5, LAMB1 and THBS3 were identified by qPCR (relative value to that of normoxia, value of normoxia assigned values of 1). Results were analyzed by unpaired Student's t-test, ${ }^{*} \mathrm{P}<0.05$ and ${ }^{* *} \mathrm{P}<0.01$ 
Table III. Numbers of alternative splicing (AS) events in 5 classes identified in HK-2 cells under hypoxic and normoxic conditions

\begin{tabular}{lcc}
\hline AS type & $\begin{array}{c}\text { HK-2 cells under } \\
\text { hypoxic conditions }(\%)\end{array}$ & $\begin{array}{c}\text { HK-2 cells under } \\
\text { normoxic conditions }(\%)\end{array}$ \\
\hline A5SS & $5,729(11.15)$ & $5,495(11.18)$ \\
A3SS & $8,290(16.13)$ & $7,898(16.07)$ \\
ES & $27,574(53.66)$ & $26,375(53.67)$ \\
IR & $4,594(8.94)$ & $4,401(8.96)$ \\
MXE & $5,200(10.12)$ & $4,975(10.12)$ \\
Total & 51,387 & 49,144 \\
\hline
\end{tabular}

A5SS, alternative 5' splice site; A3SS, alternative 3' splice site; ES, exon skipped; IR, intron retention; MXE, mutually exclusive exon; HK-2, human renal proximal tubular cells.

Table IV. Number of different types of SNPs identified by RNA sequencing.

\begin{tabular}{lrr}
\hline Sample & Hypoxic group & Normoxic group \\
\hline Total & 36,747 & 32,959 \\
Synonymous & 5,465 & 5,070 \\
Missense & 3,885 & 3,583 \\
Stopgain & 5 & 7 \\
Exonic & 9,488 & 8,788 \\
NcRNA & 2,099 & 1,859 \\
UTR5 & 1,762 & 1,642 \\
UTR5 and UTR3 & 7 & 7 \\
UTR3 & 9,564 & 8,728 \\
Intronic & 8,505 & 7,995 \\
Intergenic & 4,037 & 2,930 \\
\hline
\end{tabular}

SNPs, single nucleotide polymorphisms.

exon (MXE) (data not shown). As shown in Table III, the frequency of each AS event was highly coincident between the hypoxic group and normoxic group. Of the 5 types of AS events, ES was the most prevalent, and was comprised of $27,574(53.66 \%)$ and $26,375(53.67 \%)$. A total of 9,394 novel transcripts coding potential was found in the two samples (data not shown).

Identification of SNPS. SNPs are valuable markers that are widely used in genetics and evolution, and RNA-Seq technology has the potential to detect SNPs rapidly and reliably. To understand the changes in SNPs in HK-2 cells under hypoxic conditions, we identified a total of 69,706 SNPs (data not shown). Among these, 36,747 SNPs were from the hypoxic group and 32,959 SNPs from the normoxic group. The types of SNPs included synonymous, missense, stopgain, exonic, ncRNA, UTR5, UTR5 and UTR3, UTR3, intronic and intergenic (Table IV). The identified SNPs, as molecular markers, will be extremely helpful for the genetic linkage mapping of
HK-2 cells under hypoxic conditions. In brief, the transcriptome data will provide a valuable resource for the future development of molecular markers and functional genomics.

\section{Discussion}

Renal hypoxia is a major factor in the pathophysiology of AKI to CKD transition (3). To maintain normal metabolism, renal proximal tubular cells require a large amount of oxygen to function properly, and renal proximal tubular cells may be the primary target of a hypoxic insult (7). Although the hypoxia of HK-2 cells has been widely known in last decades, the comprehensive study of HK-2 cells under hypoxic conditions has not yet been reported, at least to the best of our knowledge. Many hypoxia-related genes have been identified in different cells, including TGF- $\beta 1$, VEGF, PFKFB4 and GLUT. The underlying molecular events however, remain unknown. Leonard et al found 48 upregulated genes in HK-2 cells exposed to hypoxia for 8,16 and $36 \mathrm{~h}$ with the aid of cDNA microarrays (19). In this study, the expression of HIF-1 $\alpha$ was increased in the hypoxic HK-2 cells compared to the nomoxic cells, which confirmed that HK-2 cells were indeed cultured in a hypoxic environment. Therefore, we then performed a comprehensive analysis of the transcriptomes of HK-2 cells under hypoxic and normoxic conditions by RNA-Seq. Compared with a previous micorray study (19), we identified a total of 279 DEGs, and 201 DEGs $(>2$-fold change) were upregulated in the HK-2 cells exposed to hypoxic conditions for $24 \mathrm{~h}$. The level and number of DEGs we obtained were not completely consistent with the previous microarry study (19). The different exposure times to hypoxia may have resulted in this discrepancy.

All DEGs were classified into GO and KEGG categories to provide an overview of their biological functions and associated biochemical pathways. Many studies have shown the expression of individual hypoxia-related genes in different cells (28-30). In the present study, our analysis indicated that the upregulated genes were highly enriched in ECM organization (GO:0030198), protein binding (GO:0005515), extra ventricular cardiac muscle cell differentiation (GO:0055012), diphosphotransferase activity (GO:0016778) and the cytoplasm (GO:0005737). Analysis for pathways revealed that ECM-receptor interaction was the most enriched upregulated pathway, including the genes related to the formation of the basement membrane. Of these genes, the COL4A2 gene encodes one of the 6 subunits of type IV collagen, the major structural component of basement membranes (31). Our data based on mRNA sequencing and qPCR confirmed the upregulated expression of subunits of type IV collagen gene under hypoxic conditions. LAMA3, LAMA5, LAMB1 belong to the laminin family for formation and function of the basement membrane (32). HSPG2 encodes the perlecan protein, which is a major component of basement membranes (33). Chronic hypoxia often occurs in the kidney tissues of many patients with CKD (18). Tubulointerstitial fibrosis leads to thickness of the tubular basement membrane and accumulation of interstitial ECM (34-37). In this study, hypoxia upregulated the expression of genes related to the basement membrane. Hypoxia upregulated the expression of matrix metalloproteinase-1 (MMP-1) and MMP-1 breaks down the interstitial collagens, types I, II and III (38). These results aid in the understanding of the 
molecular regulatory mechanisms of tubulointerstitial fibrosis and CKD.

Specifically, we found the upregulated pathway of RCC and the downregulated pathway of glutathione metabolism in HK-2 cells under hypoxic conditions. A previous study demonstrated that patients with CKD may have an increased risk of RCC (39), and another study showed that RCC may be associated with $\mathrm{CKD}$ in patients, although the mechanisms involved are unclear (40). In this study, our data suggest that hypoxia may be an important factor in promoting the development of RCC in patients with CKD. A previous study demonstrated that HIG2 protein was involved in RCC for molecular targeted therapy (41). In this study, HIG2 mRNA upregulation induced by hypoxia provides another clue for the interaction between CKD and RCC. To the best of our knowledge, this study is the first report that DEGs induced by exposure to hypoxia upregulated the pathway of RCC in HK-2 cells, as shown by RNA-seq. Glutathione may directly and indirectly participate in reactive oxygen (e.g., $\mathrm{H}_{2} \mathrm{O}_{2}$ ) through enzymatic reactions (42). Glutathione deficiency contributes to oxidative stress, which plays a key role in the pathogenesis of many diseases, including cancer, inflammation, kwashiorkor, seizure, Alzheimer's disease, Parkinson's disease, sickle cell anemia, liver disease, cystic fibrosis, attack, stroke and diabetes (43). The present study is the first to describe that hypoxia downregulated the pathway of glutathione metabolism in HK-2 cells by RNA-seq. Some studies have shown that the THBS3 gene, as a stimulator of tumor progression in osteosarcoma (44-46). This study confirmed that the expression of THBS3 may be associated with hypoxia in HK-2 cells under hypoxia by RNA-seq.

This study of the transcriptome analysis enhances our knowledge of the existing gene annotation and changes in expression patterns under hypoxic conditions, and supplements AS events, novel transcripts and SNP identification. AS is an efficient way for genomes to encode additional transcripts, and previous studies have shown that AS plays a role in source of protein diversity $(47,48)$. Novel transcripts and identification of AS patterns will contribute to a better understanding of the mechanisms of transcriptional regulation. As regards SNPs in HK-2 cells under hypoxic and normoxic conditions, their greatest importance in biomedical research is for comparing regions of the genome between cohorts in genome-wide association studies. SNPs without an observable impact on the phenotype (so-called silent mutations) are still useful as genetic markers in genome-wide association studies (49).

In conclusion, this study provides a comprehensive analysis of transcriptome in HK-2 cells under hypoxic and normoxic conditions. Comparison of the transcriptomes between two groups revealed a number of DEGs, and potential candidates involved in CKD, and further functional analysis of these genes may help us to elucidate the mechanisms responsible for the development of CKD. This study establishes a solid foundation for future genetic and functional genomics studies and expression analysis of genes in CKD.

\section{Acknowledgements}

This study was financially supported by the National Nature Science Foundation of the People's Republic of China (no. 81370868), the Fundamental Research Funds for the
Central Universities and Jiangsu Province Scientific Research Innovation Project for Graduate Students (no. KYLX_0197).

\section{References}

1. Levey AS, Atkins R, Coresh J, Cohen EP, Collins AJ, Eckardt KU, Nahas ME, Jaber BL, Jadoul M, Levin A, et al: Chronic kidney disease as a global public health problem: Approaches and initiatives - a position statement from Kidney Disease Improving Global Outcomes. Kidney Int 72: 247-259, 2007.

2. Schieppati A and Remuzzi G: Chronic renal diseases as a public health problem: Epidemiology, social, and economic implications. Kidney Int Suppl 98: S7-S10, 2005.

3. Tanaka S, Tanaka T and Nangaku M: Hypoxia as a key player in the AKI-to-CKD transition. Am J Physiol Renal Physiol 307: F1187-F1195, 2014.

4. Fine LG, Ong AC and Norman JT: Mechanisms of tubulo-interstitial injury in progressive renal diseases. Eur J Clin Invest 23: 259-265, 1993.

5. Kuncio GS, Neilson EG and Haverty T: Mechanisms of tubulointerstitial fibrosis. Kidney Int 39: 550-556, 1991.

6. Norman JT, Clark IM and Garcia PL: Hypoxia promotes fibrogenesis in human renal fibroblasts. Kidney Int 58: 2351-2366, 2000.

7. Norman JT, Orphanides C, Garcia P and Fine LG: Hypoxiainduced changes in extracellular matrix metabolism in renal cells. Exp Nephrol 7: 463-469, 1999.

8. Bunn HF and Poyton RO: Oxygen sensing and molecular adaptation to hypoxia. Physiol Rev 76: 839-885, 1996.

9. Bauer $\mathrm{C}$ and Kurtz A: Oxygen sensing in the kidney and its relation to erythropoietin production. Annu Rev Physiol 51: 845-856, 1989.

10. Pugh CW: Oxygen and genes in health and disease. QJM 90: 307-310, 1997.

11. Vaux EC, Wood SM, Cockman ME, Nicholls LG, Yeates KM, Pugh CW, Maxwell PH and Ratcliffe PJ: Selection of mutant $\mathrm{CHO}$ cells with constitutive activation of the HIF system and inactivation of the von Hippel-Lindau tumor suppressor. J Biol Chem 276: 44323-44330, 2001.

12. Semenza GL: Hypoxia-inducible factor 1 and the molecular physiology of oxygen homeostasis. J Lab Clin Med 131: 207-214, 1998.

13. Wenger RH and Gassmann M: Oxygen(es) and the hypoxiainducible factor-1. Biol Chem 378: 609-616, 1997.

14. Falanga V, Zhou L and Yufit T: Low oxygen tension stimulates collagen synthesis and COL1A1 transcription through the action of TGF-beta1. J Cell Physiol 191: 42-50, 2002.

15. Durmowicz AG, Parks WC, Hyde DM, Mecham RP and Stenmark KR: Persistence, re-expression, and induction of pulmonary arterial fibronectin, tropoelastin, and type I procollagen mRNA expression in neonatal hypoxic pulmonary hypertension. Am J Pathol 145: 1411-1420, 1994.

16. Orphanides C, Fine LG and Norman JT: Hypoxia stimulates proximal tubular cell matrix production via a TGF-beta1independent mechanism. Kidney Int 52: 637-647, 1997.

17. Tamamori $M$, Ito $H$, Hiroe $M$, Marumo $F$ and Hata RI: Stimulation of collagen synthesis in rat cardiac fibroblasts by exposure to hypoxic culture conditions and suppression of the effect by natriuretic peptides. Cell Biol Int 21: 175-180, 1997.

18. Fine LG and Norman JT: Chronic hypoxia as a mechanism of progression of chronic kidney diseases: From hypothesis to novel therapeutics. Kidney Int 74: 867-872, 2008.

19. Leonard MO, Cottell DC, Godson C, Brady HR and Taylor CT: The role of HIF-1 alpha in transcriptional regulation of the proximal tubular epithelial cell response to hypoxia. J Biol Chem 278: 40296-40304, 2003.

20. Ulbrich SE: Groebner AE and Bauersachs S: Transcriptional profiling to address molecular determinants of endometrial receptivity - lessons from studies inlivestock species. Methods 59: 108-115, 2013.

21. Lahr DJ, Grant JR and Katz LA: Multigene phylogenetic reconstruction of the Tubulinea (Amoebozoa) corroborates four of the six major lineages, while additionally revealing that shell composition does not predict phylogeny in the Arcellinida. Protist 164: 323-339, 2013.

22. Carrara M, Lum J, Cordero F, Beccuti M, Poidinger M, Donatelli S, Calogero RA and Zolezzi F: Alternative splicing detection workflow needs a careful combination of sample prep and bioinformatics analysis. BMC Bioinformatics 16 (Suppl 9): S2, 2015. 
23. Trapnell C, Pachter L and Salzberg SL: TopHat: Discovering splice junctions with RNA-Seq. Bioinformatics 25: 1105-1111, 2009.

24. Trapnell C, Williams BA, Pertea G, Mortazavi A, Kwan G, van Baren MJ, Salzberg SL,Wold BJ and Pachter L: Transcript assembly and quantification by RNA-Seq reveals unannotated transcripts and isoform switching during cell differentiation. Nat Biotechnol 28: 511-515, 2010.

25. Katz Y, Wang ET, Airoldi EM and Burge CB: Analysis and design of RNA sequencing experiments for identifying isoform regulation. Nat Methods 7: 1009-1015, 2010.

26. McKenna A, Hanna M, Banks E, Sivachenko A, Cibulskis K, Kernytsky A, Garimella K, Altshuler D, Gabriel S, Daly M and DePristo MA: The Genome Analysis Toolkit: A MapReduce framework for analyzing next-generation DNA sequencing data. Genome Res 20: 1297-1303, 2010.

27. DePristo MA, Banks E, Poplin R, Garimella KV, Maguire JR, Hartl C, Philippakis AA, del Angel G, Rivas MA, Hanna M, et al: A framework for variation discovery and genotyping using nextgeneration DNA sequencing data. Nat Genet 43: 491-498, 2011.

28. Zscharnack K, Kessler R and Bleichert F: Warnke JP and Eschrich K: The PFKFB3 splice variant UBI2K4 is downregulated in high-grade astrocytomas and impedes the growth of U87glioblastoma cells. Neuropathol Appl Neurobiol 35: 566-578, 2009 .

29. Starska K, Forma E, Jóźwiak P, Bryś M, Lewy-Trenda I, Brzezińska-Błaszczyk E and Krześlak A: Gene and protein expression of glucose transporter 1 and glucose transporter 3 in human laryngeal cancer-the relationship with regulatory hypoxiainducible factor- $1 \alpha$ expression, tumor invasiveness, and patient prognosis. Tumour Biol 36: 2309-2321, 2015.

30. Hu JW, Sun P, Zhang DX, Xiong WJ and Mi J: Hexokinase 2 regulates G1/S checkpoint through CDK2 in cancer-associated fibroblasts. Cell Signal 26: 2210-2216, 2014.

31. Kalluri R and Sukhatme VP: Fibrosis and angiogenesis. Curr Opin Nephrol Hypertens 9: 413-418, 2000.

32. Lee SM, Chung M, Hwang KJ, Ju YR, Hyeon JW, Park JS, Kim CK, Choi S, Lee J and Kim SY: Biological network inferences for a protection mechanism against familial Creutzfeldt-Jakob disease with E200K pathogenic mutation. BMC Med Genomics 7: 52, 2014.

33. Warren CR, Grindel BJ and Francis L: Carson Dd and Farach-Carson MC: Transcriptional activation by NFאB increases perlecan/HSPG2 expression in the desmoplastic prostate tumormicroenvironment. J Cell Biochem 115: 1322-1333, 2014

34. Jacobson HR: Chronic renal failure: Pathophysiology. Lancet 338: 419-423, 1991

35. Luo X, Deng L, Lamsal LP, Xu W, Xiang C and Cheng L: AMP-activated protein kinase alleviates extracellular matrix accumulation in high glucose-induced renal fibroblasts through mTOR signaling pathway. Cell Physiol Biochem 35: 191-200, 2015.
36. Zhou X, Zhang J, Xu C and Wang W: Curcumin ameliorates renal fibrosis by inhibiting local fibroblast proliferation and extracellular matrix deposition. J Pharmacol Sci 126: 344-350, 2014.

37. Eddy AA: Molecular insights into renal interstitial fibrosis. J Am Soc Nephrol 7: 2495-2508, 1996.

38. Huang SF, Li YH, Ren YJ, Cao ZG and Long X: The effect of a single nucleotide polymorphism in the matrix metalloproteinase-1 (MMP-1) promoter on force-induced MMP-1 expression in human periodontal ligament cells. Eur J Oral Sci 116: 319-323, 2008.

39. Hofmann JN and Purdue MP: CKD and risk of renal cell carcinoma: A causal association? J Am Soc Nephrol 25: 2147-2148, 2014.

40. Kim YW, Kim WT, Yun SJ, Lee SC, Kim WJ, Ha YS, Park YH, Kang SH, Hong SH, Kwon TG, et al: Preoperative chronic kidney disease status is an independent prognostic factor in patients with renal cell carcinoma. Ann Surg Oncol 22: 4098-4103, 2015.

41. Togashi A, Katagiri T, Ashida S, Fujioka T, Maruyama O, Wakumoto Y, Sakamoto Y, Fujime M, Kawachi Y, Shuin T and Nakamura Y: Hypoxia-inducible protein 2 (HIG2), a novel diagnostic marker for renal cell carcinoma and potential target for molecular therapy. Cancer Res 65: 4817-4826, 2005.

42. Fang YZ, Yang S and Wu G: Free radicals, antioxidants, and nutrition. Nutrition 18: 872-879, 2002.

43. Wu G, Fang YZ, Yang S, Lupton JR and Turner ND: Glutathione metabolism and its implications for health. J Nutr 134: 489-492, 2004.

44. Straume O and Akslen LA: Expresson of vascular endothelial growth factor, its receptors (FLT-1, KDR) and TSP-1 related to microvessel density and patient outcome in vertical growth phase melanomas. Am J Pathol 159: 223-235, 2001.

45. Qian X and Tuszynski GP: Expression of thrombospondin-1 in cancer: A role in tumor progression. Proc Soc Exp Biol Med 212: 199-207, 1996.

46. Chandrasekaran L, He CZ, Al-Barazi H and Krutzsch HC: Iruela-Arispe Ml and Roberts DD: Cell contact-dependent activation of alpha3betal integrin modulates endothelial cell responses tothrombospondin-1. Mol Biol Cell 11: 2885-2900, 2000.

47. Brett D, Pospisil H, Valcárcel J, Reich J and Bork P: Alternative splicing and genome complexity. Nat Genet 30: 29-30, 2002.

48. Li HD, Menon R, Omenn GS and Guan Y: The emerging era of genomic data integration for analyzing splice isoform function. Trends Genet 30: 340-347, 2014.

49. Thomas PE, Klinger R, Furlong LI, Hofmann-Apitius M and Friedrich CM: Challenges in the association of human single nucleotide polymorphism mentions with unique database identifiers. BMC Bioinformatics 12 (Suppl 4): S4, 2011 